\title{
Combination Cancer Immunotherapy with Molecular Targeted Agents/Anti-CTLA-4 Antibody for Hepatocellular Carcinoma
}

\author{
Masatoshi Kudo \\ Department of Gastroenterology and Hepatology, Kindai University Faculty of Medicine, \\ Osaka-Sayama, Japan
}

\section{Introduction}

Prof. M. Kudo

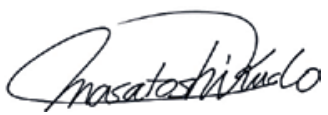

Editor Liver Cancer

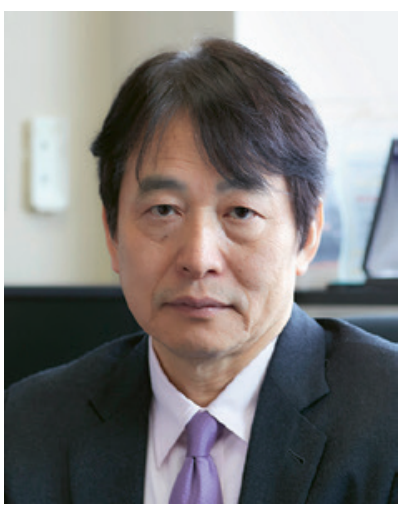

The recent development of immunotherapy has advanced the treatment of various cancers. The anti-PD-1 antibody nivolumab was designated by the FDA as a breakthrough therapy in September 2014. Subsequently, the anti-PD-1 antibody pembrolizumab received the same designation. Nivolumab is currently approved as a drug with good efficacy against a range of specific malignancies including malignant melanoma, non-small cell lung cancer, kidney cancer, stomach cancer, Hodgkin's lymphoma, and urothelial carcinoma. In addition, it is being investigated for the treatment of other types of cancer in promising clinical studies. Pembrolizumab was also approved by the FDA for similar indications in addition to the microsatellite instability-high or mismatch repair-deficient solid tumors.

Hepatocellular carcinoma (HCC) is considerably more heterogeneous than other solid tumors or hematologic malignancies. Because of the lack of major driver mutations in HCC, treating patients with drugs that deplete hepatic functional reserve is challenging. Accordingly, researchers are investigating therapeutic approaches that differ from those used for other cancers. Among such therapies, the anti-PD-1 antibody nivolumab showed promise as a therapeutic agent for HCC in the CheckMate040 study [1]. Furthermore, numerous pharmaceutical companies have initiated phase III and/or earlier clinical trials of anti-PD-1 or antiPD-L1 antibodies and anti-CTLA-4 antibodies for HCC (Table 1). 
Table 1. Immune checkpoint inhibitors

\begin{tabular}{|c|c|c|c|c|c|c|}
\hline Target cell & Molecule & Develop. code & Drug name & TM name & Antibody class & Company \\
\hline \multirow[t]{4}{*}{$\mathrm{T}$ cell } & PD-1 & $\begin{array}{l}\text { BMS-36558 } \\
\text { ONO-4538 }\end{array}$ & Nivolumab & Opdivo & $\begin{array}{l}\text { Fully human } \\
\text { IgG4 antibody }\end{array}$ & ONO/BMS \\
\hline & PD-1 & MK-4375 & Pembrolizumab & Keytruda & $\begin{array}{l}\text { Humanized } \\
\text { IgG4 antibody }\end{array}$ & Merck \\
\hline & PD-1 & BGB-A317 & Tislelizumab & Not approved & $\begin{array}{l}\text { Humanized } \\
\text { IgG4 antibody }\end{array}$ & BeiGene \\
\hline & PD-1 & SHR-1210 & Camrelizumab & Not approved & $\begin{array}{l}\text { Humanized } \\
\text { IgG4 antibody }\end{array}$ & $\begin{array}{l}\text { Jiangsu } \\
\text { HengRui } \\
\text { Medicine }\end{array}$ \\
\hline \multirow[t]{3}{*}{ Tumor cell } & PD-L1 & MPDL3280A & Atezolizumab & Tecentriq & $\begin{array}{l}\text { Fully human } \\
\text { IgG1 antibody }\end{array}$ & Roche \\
\hline & PD-L1 & MEDI4736 & Durvalumab & Imfinzi & $\begin{array}{l}\text { humanized } \\
\text { IgG1 antibody }\end{array}$ & AstraZeneca \\
\hline & PD-L1 & MSB-0010718C & Avelumab & Barencio & $\begin{array}{l}\text { Humanized } \\
\text { IgG1 antibody }\end{array}$ & Merck-Serono \\
\hline \multirow[t]{2}{*}{$\mathrm{T}$ cell } & CTLA-4 & BMS-734016 & Ipilimumab & Yervoy & $\begin{array}{l}\text { Fully humanized } \\
\text { IgG1 antibody }\end{array}$ & $\begin{array}{l}\text { BMS } \\
\text { Medarex }\end{array}$ \\
\hline & CTLA-4 & MEDI1123 & Tremelimumab & Not approved & $\begin{array}{l}\text { Fully humanized } \\
\text { IgG2 antibody }\end{array}$ & $\begin{array}{l}\text { AstraZeneca } \\
\text { MedImmune }\end{array}$ \\
\hline
\end{tabular}

Single-agent HCC therapies with anti-PD-1 antibody have yielded a higher response rates ranging from 17 to $20 \%$ with durable response than previously reported molecular targeted agents (MTAs) [1, 2]. Therefore, nivolumab was approved in September 22, 2017, as secondline therapy for HCC patients after progression on sorafenib therapy based on the phase I/II study only. For similar reasons, pembrolizumab was also approved by the FDA (November 9 , 2018) as second-line therapy for patients with HCC who progress after sorafenib therapy.

However, these agents show limited benefit from monotherapy of immune checkpoint inhibitors in approximately $30-40 \%$ of patients with progressive disease or those with stable disease developing into progressive disease. Researchers are therefore actively seeking therapeutic approaches for HCC that combine immune checkpoint inhibitors and other agents, similar to the therapeutic strategies commonly used in other cancers. Representative examples include immune checkpoint inhibitor combinations (PD-1/PD-L1 antibodies plus CTLA-4 antibodies), PD-1/PD-L1 antibodies plus MTAs, and PD-1/PD-L1 antibodies plus existing local therapeutic agents (Fig. 1).

\section{Immune Checkpoint Inhibitor Combination Therapies}

In the field of immunotherapy, the results of combination therapy with anti-PD-1/antiPD-L1 antibodies and anti-CTLA-4 antibodies in HCC are awaited with much anticipation (Fig. 1). Concomitant nivolumab and ipilimumab show a superior therapeutic effect over monotherapies for melanoma [3, 4], although the efficacy of this combination in HCC is under investigation $[5,6]$. The rationale for combination therapies with anti-PD-1/anti-PD-L1 and 


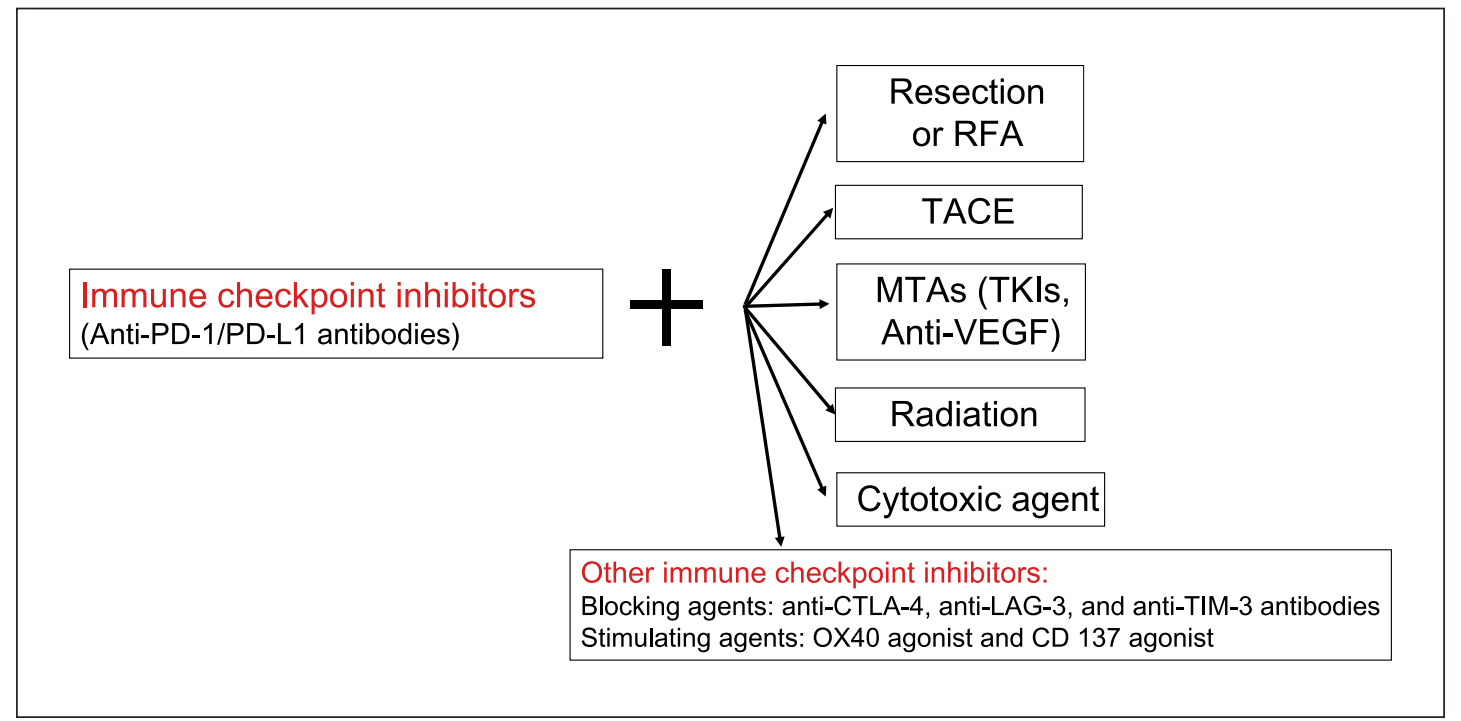

Fig. 1. Treatment strategy using immune checkpoint inhibitors. Future direction: combination therapy. RFA, radiofrequency ablation; TACE, transarterial chemoembolization; MTAs, molecular targeted agents; TKIs, tyrosine kinase inhibitors; VEGF, vascular endothelial growth factor.

\section{The Cancer-Immunity Cycle}

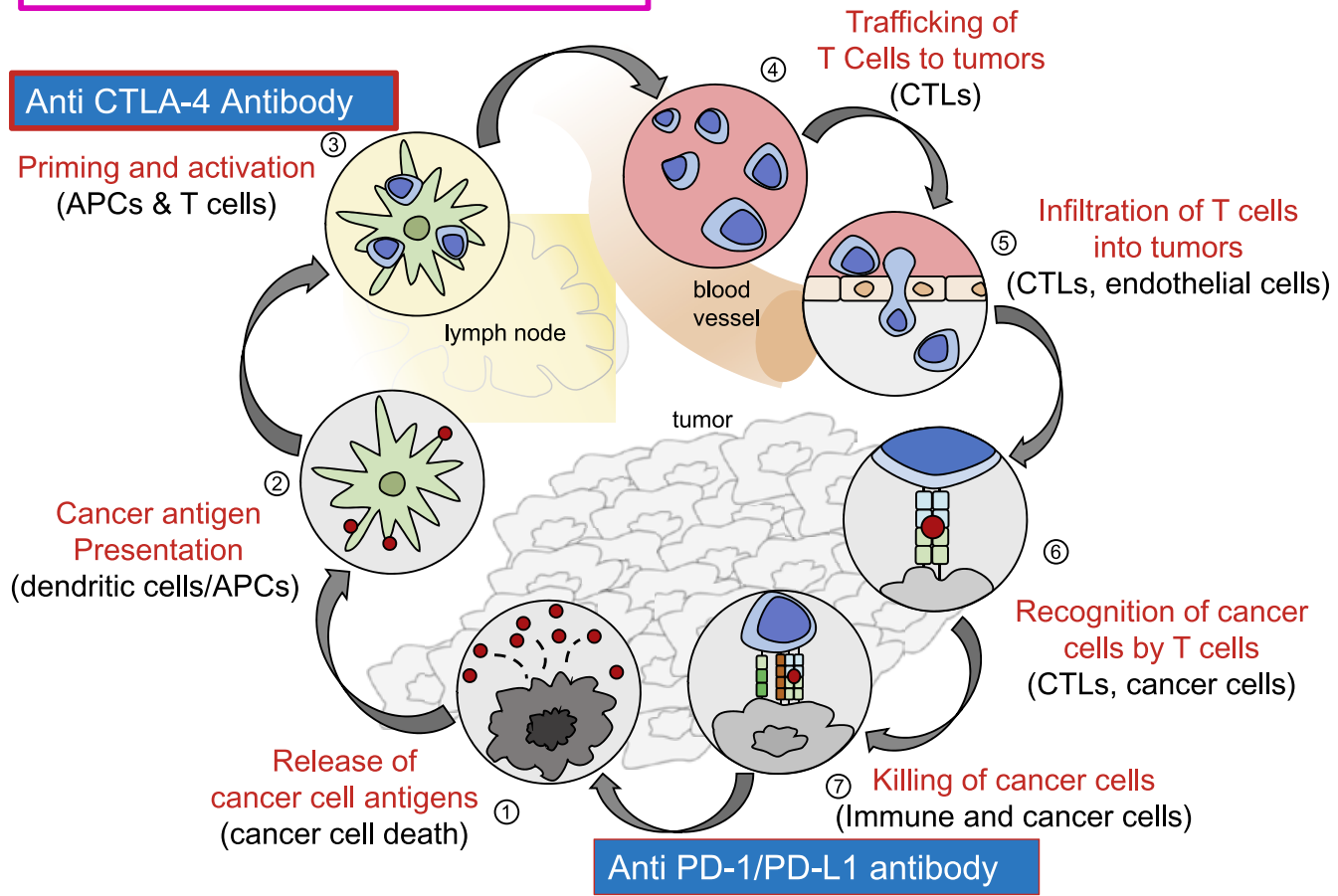

Fig. 2. The cancer immunity cycle. Modified from Chen and Mellman [7]. 


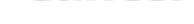

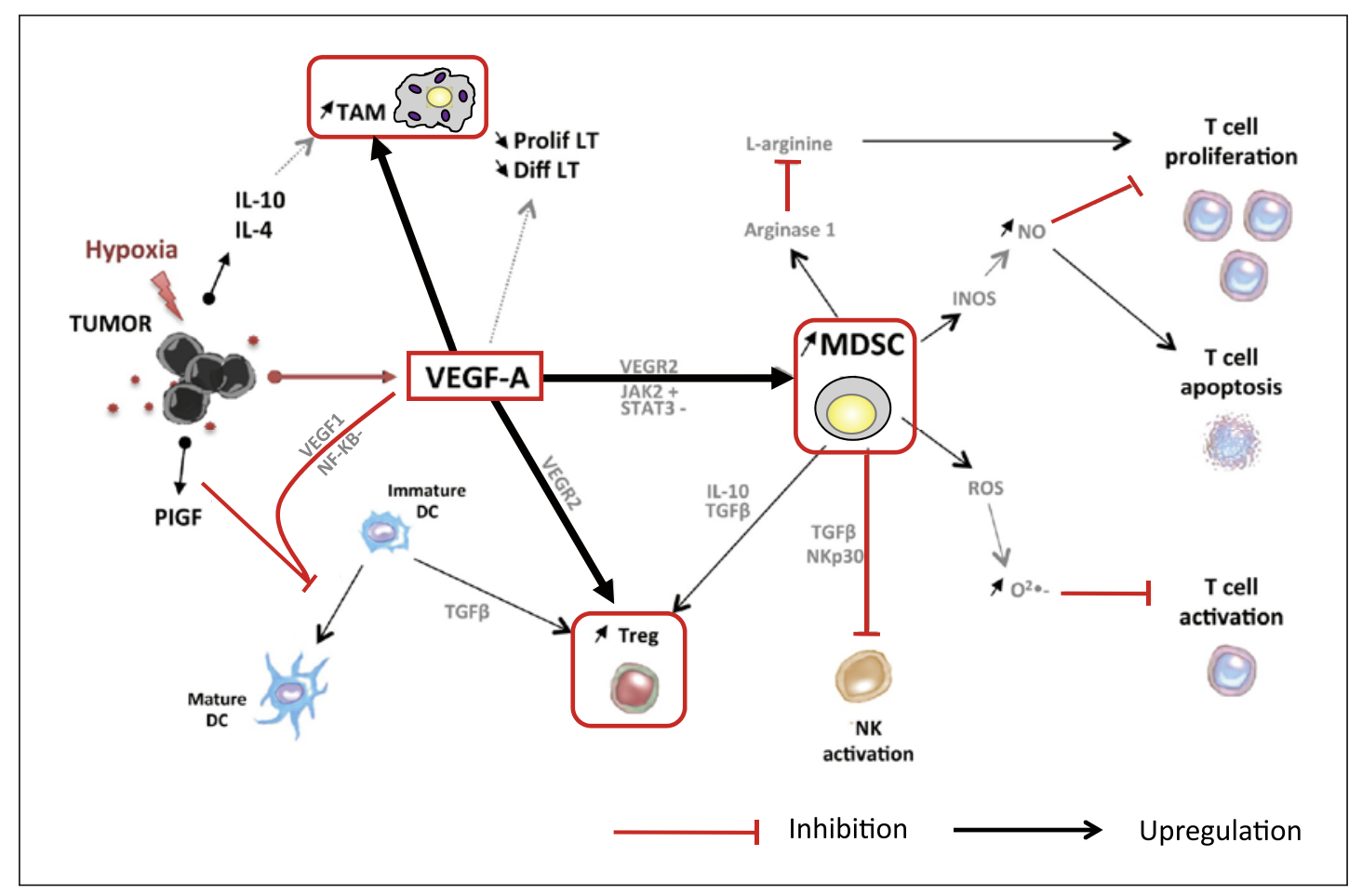

Fig. 3. Immunosuppressive microenvironment produced by VEGF. MDSC, myeloid-derived suppressor cell; DC, dendritic cell; TAM, tumor-associated macrophage; T-reg, T-regulatory cell. Modified from Voron et al. [9].

anti-CTLA-4 antibodies is as follows: In the absence of $\mathrm{CD}^{+} \mathrm{T}$ cells, stimulation of tumor immunity cannot be expected because these essential lymphocytes are absent from the tumor site, regardless of whether the PD-1/PD-L1 pathway is blocked. However, the anti-tumor effect is increased by the concomitant administration of anti-CTLA- 4 antibodies that block the B7-CTLA-4 pathway, as this block leads to an increase in activated CD8 ${ }^{+} \mathrm{T}$ cells in lymph nodes followed by an increase in activated $\mathrm{CD}^{+} \mathrm{T}$ cells infiltrating the tumor tissue [7] (Fig. 2). Furthermore, CTLA-4 is expressed on regulatory T cells ( $\mathrm{T}_{\text {reg }}$ cells) that infiltrate into tumors to play a role as suppressors of anti-tumor immunity in the tumor microenvironment [8]. Theoretically, anti-CTLA-4 antibodies should enhance $\mathrm{CD}^{+} \mathrm{T}$ cell immune activation by inhibiting CTLA-4 expressed on $\mathrm{T}_{\text {reg }}$ cells. This is the rationale for the ongoing clinical trials of combination therapies of anti-CTLA-4 antibodies with anti-PD-1/PD-L1 antibodies for HCC (Fig. 2).

Combination therapy with nivolumab and the anti-CTLA-4 antibody ipilimumab at a range of doses and dose intervals is under investigation in the CheckMate 040 study. Another therapeutic regimen currently under investigation is the combination of the anti-PD-L1 antibody durvalumab and the anti-CTLA-4 antibody tremelimumab, and the efficacy and safety of this combination are being evaluated in comparison with monotherapies in a phase I/II clinical study. The phase I part of this study was reported at the 2017 annual meeting of the American Society of Clinical Oncology (ASCO) [5]. Durvalumab plus tremelimumab combination therapy showed an objective response rate (ORR) of $25 \%$ in 40 HCC patients, yielding a more favorable outcome than durvalumab monotherapy [5]. These results were the basis for the initiation of a currently ongoing phase III clinical study (HIMALAYA study). 


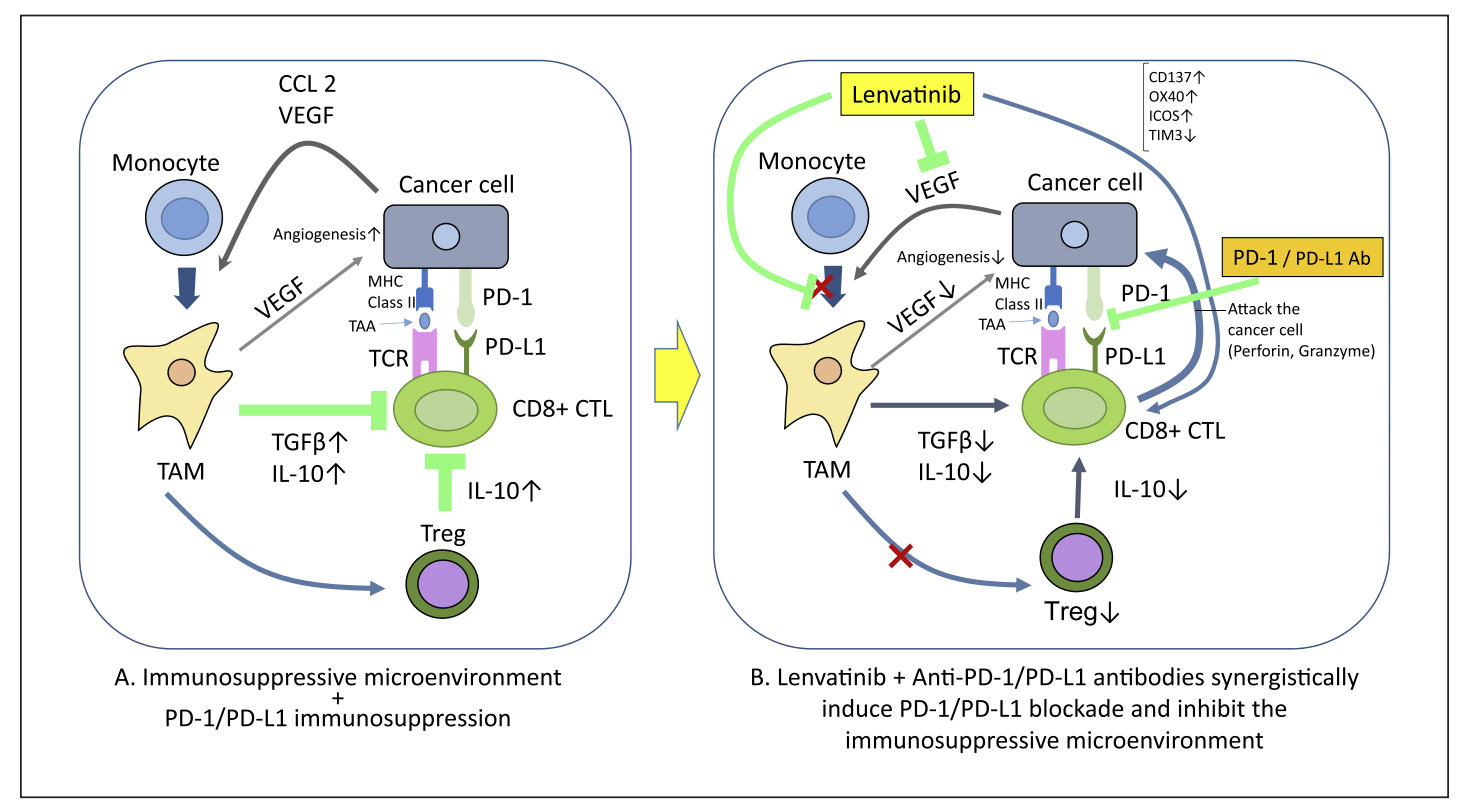

Fig. 4. Mechanism of synergistic effects of lenvatinib and anti-PD-1/PD-L1 antibodies.

\section{Combination Therapies with Immune Checkpoint Inhibitors and MTAs}

Immune checkpoint inhibitor-MTA combination therapies have raised high expectations in recent years. These therapies affect vascular endothelial growth factor (VEGF)-A, which is released from tumor cells and the vascular endothelium in the region of the cancer. VEGF-A induces a wide range of immune suppressor cells, including tumor-associated macrophages (TAMs), $\mathrm{T}_{\text {reg }}$ cells, and myeloid-derived suppressor cells (MDSCs), creating a microenvironment that suppresses tumor immunity (Fig. 3). These cells prevent the maturation of dendritic cells and inhibit the activation of NK cells and T cells by releasing immunosuppressive cytokines (IL-10 or TGF- $\beta$ ) [9]. Stromal cells in the liver (e.g., Kupffer cells, dendritic cells, hepatic endothelial cells, and hepatic stellate cells) are also implicated in the immunosuppressive environment of HCC [10]. On this theoretical basis, VEGF activity could be suppressed by anti-VEGF antibodies and multikinase (including VEGF)-inhibiting MTAs in the following ways: (1) antigen presentation to dendritic cells is increased; (2) T cell activation is promoted in the priming phase; (3) tumor vessels are normalized, thereby improving trafficking and infiltration of T cells from lymph nodes to the region of the tumor; (4) the $\mathrm{T}_{\text {reg }}$ cells, TAMs, and MDSCs mentioned above are suppressed, and the humoral factors TGF- $\beta$ and IL-10 decrease at the tumor site, leading to improved immunity in the tumor microenvironment; (5) the inhibition of checkpoint molecules by anti-PD-1/PD-L1 antibodies activates the cancer immunity cycle against the tumor [7]. Furthermore, activated T cells can then effectively attack the tumor, inducing necrosis of tumor cells (Fig. 2). Pembrolizumab and lenvatinib combination therapy suppresses TAMs and $\mathrm{T}_{\text {reg }}$ cells, counteracting their immunosuppressive effect on the microenvironment. This decreases TGF- $\beta$ and IL-10, downregulates the expression of PD-1 and T cell immunoglobulin and mucin domain 3 (TIM-3), and promotes the immune-activating cytokine IL-12, resulting in an anti-tumor immunity-promoting effect, as shown in a preclinical study [11] (Fig. 4). A number of clinical trials of immune checkpoint inhibitor-MTA combination therapies have been initiated based on this hypothesis (Table 2). Pembrolizumab plus lenvatinib combination therapy shows high response rates of $50-70 \%$ 
Table 2. Immune checkpoint inhibitors in combination with MTAs in HCC

\begin{tabular}{|c|c|c|c|c|}
\hline Phase & Target & Agent & Company & Trial \# \\
\hline $1-2$ & PD-1 + TGF $\beta$ receptor I & Nivolumab + galunisertib & Eli Lilly & NCT02423343 \\
\hline 1 & PD-L1 + VEGFR-2 & Durvalumab + ramucirumab & Eli Lilly & NCT02572687 \\
\hline 1 & PD-1 + multikinase & Pembrolizumab + nintedanib & $\begin{array}{l}\text { Gustave } \\
\text { Roussy }\end{array}$ & NCT02856425 \\
\hline 1 & PD-1 + multikinase & PDR001 + sorafenib & Novartis & NCT02988440 \\
\hline $1-2$ & PD-1 + c-Met & PDR001 + capmatinib (INC280) & Novartis & NCT02795429 \\
\hline $1-2$ & PD-1; CTLA-4 + MET/VEGFR2 & Nivolumab + ipilimumab + cabozantinib & BMS & NCT01658878 \\
\hline 1 & PD-1 + multikinase & Nivolumab + lenvatinib & Ono & NCT03418922 \\
\hline $1-2$ & PD-L1 + multikinase & Averumab + axitinib & Pfizer & NCT03289533 \\
\hline 1 & PD-1 + multikinase & SHR-1210 + apatinib & $\begin{array}{l}\text { Jiangsu } \\
\text { HengRui } \\
\text { Medicine }\end{array}$ & NCT02942329 \\
\hline 1 & PD-1 + multikinase & Pembrolizumab + lenvatinib & Eisai & NCT03006926 \\
\hline $1-2$ & PD-L1 + anti-VEGF & Atezolizumab + bevacizumab & Roche & NCT03170960 \\
\hline 3 & PD-L1 + anti-VEGF & Atezolizumab + bevacizumab & Roche & NCT03434379 \\
\hline
\end{tabular}

and durable responses in renal cancer, endometrial cancer, and head and neck cancer according to results reported at the 2018 annual meeting of the European Society of Medical Oncology (ESMO), and the 2017 and 2018 ASCO meetings. A number of similar, early-phase clinical studies of HCC therapies are currently ongoing, including the pembrolizumab plus lenvatinib trial and atezolizumab plus bevacizumab trial (Table 2).

The results of three anti-PD-1/PD-L1 antibody plus MTA combination immunotherapies for HCC were presented simultaneously at the 2018 annual ASCO meeting. The first of these reports involved a phase $\mathrm{Ib}$ clinical trial of pembrolizumab plus lenvatinib combination therapy. In that study, safety and efficacy evaluations included only 30 and 26 patients, respectively; however, the ORR was $42.3 \%$, and the disease control rate was $100 \%$, with no cases of progressive disease. Outcomes were favorable, and progression-free survival was 9.7 months [12] (Table 3).

The second of these reports involved combination therapy with the anti-PD-L1 antibody atezolizumab and the anti-VEGF antibody bevacizumab. The results generated considerable interest in the field of HCC therapy because of the high ORR of $61 \%$ based on RECIST 1.1 and according to an investigator assessment of 23 patients, and $65 \%$ according to an independent review [13].

The third of these reports involved the anti-PD-1 antibody SHR1210 combined with apatinib. This study involved 18 patients and yielded fair outcomes, with a response rate of $38.9 \%$, disease control rate of $83.3 \%$, and progression-free survival of 7.2 months [14] (Table 3).

The highest response rate reported at ASCO 2018 in June was for the clinical study of atezolizumab plus bevacizumab. However, updated results (including 73 patients) were reported at the 2018 annual ESMO meeting in October, and the response rates were substantially reduced: $32 \%$ according to RECIST 1.1 by investigator assessment and $27 \%$ according to RECIST 1.1 by independent assessment [15] (Tables 4, 5). The ORR of $27 \%$ according to independent review that was reported at ESMO 2018 was greatly reduced from the corre- 
Kudo: Combination Cancer Immunotherapy with Molecular Targeted

Agents/Anti-CTLA-4 Antibody for Hepatocellular Carcinoma

Table 3. Results of immune checkpoint inhibitors and combination therapy

\begin{tabular}{|c|c|c|c|c|c|c|}
\hline & $\begin{array}{l}\text { Nivolumab } \\
(n=214)\end{array}$ & $\begin{array}{l}\text { Pembrolizumab } \\
(n=104)\end{array}$ & $\begin{array}{l}\text { Pembrolizumab } \\
\text { plus } \\
\text { lenvatinib } \\
(n=26)\end{array}$ & $\begin{array}{l}\text { Atezolizumab } \\
\text { plus } \\
\text { bevacizumab } \\
(n=73)\end{array}$ & $\begin{array}{l}\text { SHR-1210 } \\
\text { plus } \\
\text { apatinib } \\
(n=18)\end{array}$ & $\begin{array}{l}\text { Durvalumab } \\
\text { plus } \\
\text { tremelimumab } \\
(n=40)\end{array}$ \\
\hline ORR, \% & $20(15-26)^{b}$ & $17(11-26)^{b}$ & $42.3(23.4-63.1)^{c}$ & $34^{\mathrm{c}}$ & $38.9^{c}$ & $25^{b}$ \\
\hline DCR, \% & $64(58-71)$ & $62(52-71)$ & 100 & 75 & 83.3 & 57.5 (>16 weeks) \\
\hline PFS, months & $4.0(2.9-5.4)$ & $4.9(3.4-7.2)$ & 9.7 (5.6-NE) & $7.5(0.4-23.9)$ & $7.2(2.6-\mathrm{NE})$ & NA \\
\hline OS, months & NR (9 M, 74\%) & $12.9(9.7-15.5)$ & NR & NR & NR & NA \\
\hline DOR, months & $9.9(8.3-\mathrm{NE})$ & $\leq 9(77 \%)$ & $\mathrm{NE}$ & NR & $\mathrm{NE}$ & NA \\
\hline
\end{tabular}

Figures in parentheses indicate 95\% CI unless otherwise stated. ORR, objective response rate; DCR, disease control rate; PFS, progression-free survival; OS, overall survival; DOR, duration of response; NR, not reached; NE, not estimable; NA, not available. ${ }^{a}$ Independent Review Facility (IRF) assessment. ${ }^{\mathrm{b}}$ RECIST $1.1 .{ }^{\mathrm{c}}$ Modified RECIST.

Table 4. Comparison between data at ASCO 2018 and ESMO 2018 [15]

\begin{tabular}{lcc}
\hline & $\begin{array}{c}\text { ASCO } 2018 \\
\text { Atezo }+ \text { Bev }(n=23)\end{array}$ & $\begin{array}{l}\text { ESMO 2018 } \\
\text { Atezo }+\operatorname{Bev}(n=73)\end{array}$ \\
\hline ORR, $n(\%)$ & $14(61)$ & $23(32)$ \\
CR & $0(0)$ & $1(1)$ \\
PR & $14(61)$ & $22(30)$ \\
SD & $5(22)$ & $33(45)$ \\
PD & $4(17)$ & $13(18)$ \\
DCR (CR + PR + SD), $n(\%)$ & $19(83)$ & $56(78)$ \\
\hline
\end{tabular}

Investigator-assessed results (per RECIST v1.1). Seventy-three patients were evaluable for efficacy with a minimum followup of 16 weeks. ORR, objective response rate; CR, complete response; PR, partial response; SD, stable disease; PD, progressive disease; DCR, disease control rate.

Table 5. Comparison between data at ASCO 2018 and ESMO 2018 [15]

\begin{tabular}{|c|c|c|c|}
\hline & $\begin{array}{l}\text { ASCO } 2018 \\
\text { Atezo }+ \text { Bev }(n=23)^{a}\end{array}$ & $\begin{array}{l}\text { ESMO } 2018 \\
\text { Atezo }+\operatorname{Bev}(n=73)^{\mathrm{a}}\end{array}$ & $\begin{array}{l}\text { ESMO } 2018 \\
\text { Atezo }+ \text { Bev }(n=73)^{b}\end{array}$ \\
\hline ORR, $n(\%)$ & $15(65)$ & 20 (27) & $25(34)$ \\
\hline $\mathrm{CR}$ & $1(4)$ & $4(5)$ & $8(11)$ \\
\hline PR & $14(61)$ & $16(22)$ & $17(23)$ \\
\hline SD & $7(30)$ & $35(48)$ & $30(41)$ \\
\hline PD & $1(4)$ & 14 (19) & 14 (19) \\
\hline $\mathrm{DCR}(\mathrm{CR}+\mathrm{PR}+\mathrm{SD}), n(\%)$ & $22(96)$ & $55(75)$ & $55(75)$ \\
\hline
\end{tabular}

Seventy-three patients were evaluable for efficacy with a minimum follow-up of 16 weeks. ORR, objective response rate; CR, complete response; PR, partial response; SD, stable disease; PD, progressive disease; DCR, disease control rate. ${ }^{\text {a }}$ Independent review facility-assessed results (per RECIST v1.1). ${ }^{\mathrm{b}}$ Independent review facility-assessed results (per mRECIST).

sponding ORR of $65 \%$ reported at ASCO 2018. The ORR of this combination trial was $34 \%$ according to the modified RECIST by independent review assessment (Table 5).

A major factor in the reduction of response rate was the increase in the proportion of patients with macrovascular invasion (MVI) and/or extrahepatic spread (EHS) from 65\% (ASCO 2018) to 88\% (ESMO 2018) (Table 6; Fig. 5). The ESMO data showed that the response 
Kudo: Combination Cancer Immunotherapy with Molecular Targeted

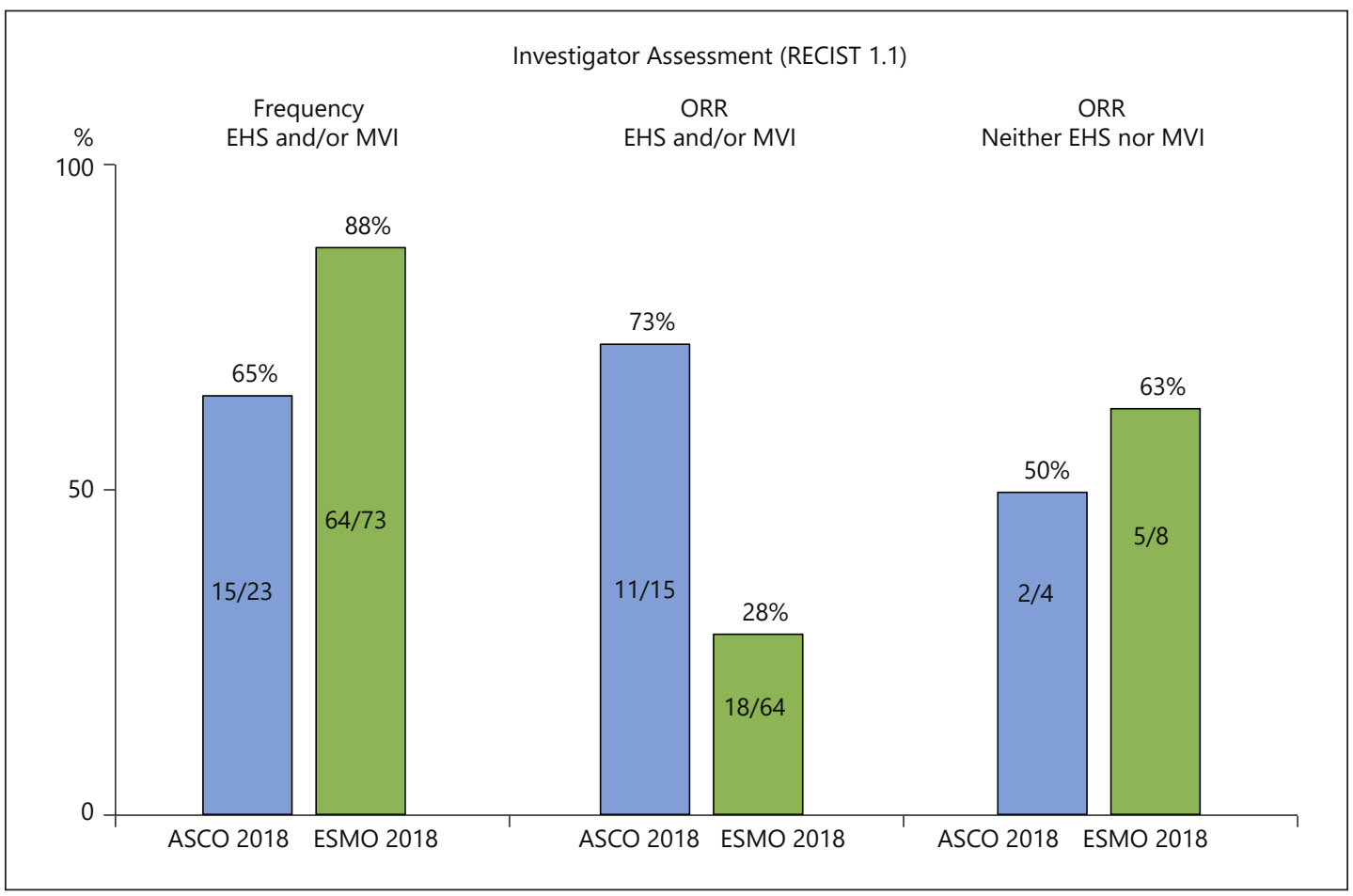

Fig. 5. Phase $1 \mathrm{~b}$ of atezolizumab + bevacizumab combination therapy. Extrahepatic spread (EHS) and/or macrovascular invasion (MVI).

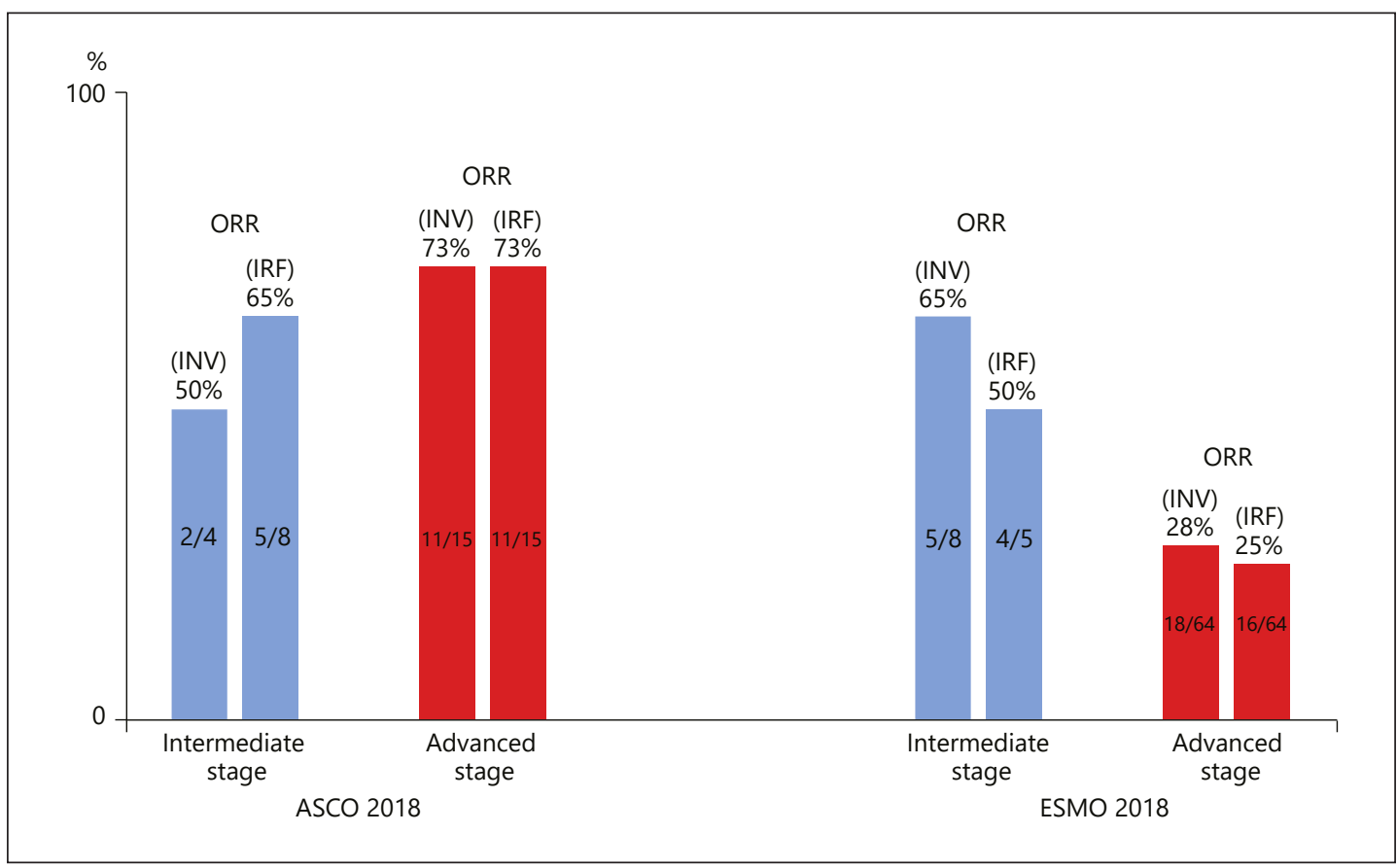

Fig. 6. Phase $1 \mathrm{~b}$ of atezolizumab + bevacizumab combination therapy. Comparison of ORR between intermediate and advanced HCC. ORR, objective response rate; INV, investigator assessment; IRF, independent review facility assessment. Intermediate stage: neither extrahepatic spread nor macrovascular invasion. Advanced stage: extrahepatic spread and/or macrovascular invasion. 


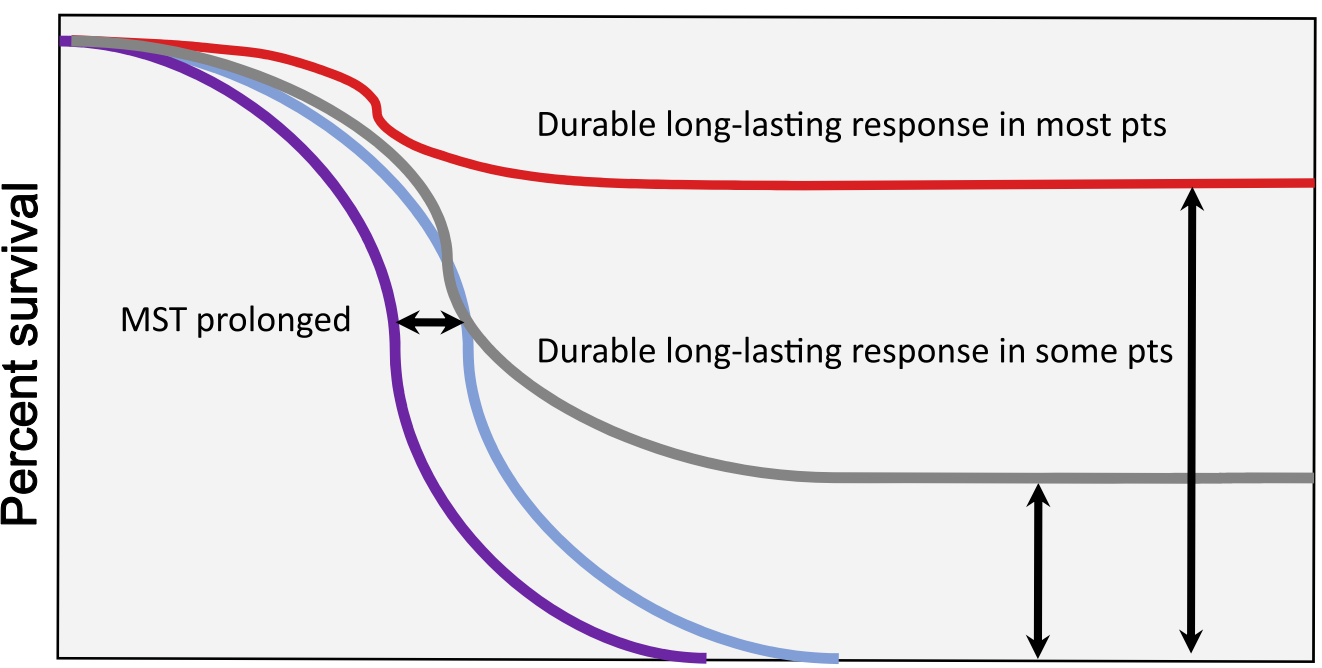

Time

\section{Chemotherapy \\ Molecular targeted therapy \\ Immune checkpoint therapy}

(c) 2019 S. Karger AG, Basel

www.karger.com/lic

Kudo: Combination Cancer Immunotherapy with Molecular Targeted

Agents/Anti-CTLA-4 Antibody for Hepatocellular Carcinoma

Fig. 7. Improved overall survival as a result of combination therapy: immune checkpoint inhibitors and molecular targeted therapy. Modified from Sharma and Allison [18].

rate was 28\% in patients with MVI and/or EHS (advanced stage HCC), compared with 63\% in patients with neither MVI nor EHS (intermediate stage HCC). Advanced-stage HCC is a subgroup that responds poorly to systemic therapy compared with intermediate-stage HCC (Table 6; Fig. 6). According to the ASCO results, the response rate was $73 \%$ in patients with MVI and/or EHS (advanced stage HCC) versus 50\% in patients with neither MVI nor EHS (intermediate stage HCC). These figures show an opposite pattern to that of the ESMO results (Fig. 6). The ORR reported at ESMO 2018 appears to be consistent with the pattern in the previous systemic therapy, as the ORR of a Japanese subpopulation from the REFLECT trial was $61.3 \%(n=31)$ for intermediate-stage HCC and 38.0\% $(n=50)$ for advanced-stage HCC in the lenvatinib arm [16].

It is easily speculated that progressive disease is more frequently observed, and tumor shrinkage is more difficult to achieve in advanced-stage HCC because of the more frequently observed phenotype change, such as epithelial-mesenchymal transition, and/or higher tumor burden/vascular invasion than in intermediate-stage HCC. The higher response rates in advanced-stage HCC than those in intermediate-stage HCC reported at ASCO 2018 could be attributed to the smaller number of patients evaluated.

The currently available results for combination immunotherapies are shown in Table 3. It may be important to note that the ORR may change, either improve or decrease, with further increases in the number of patients evaluated from an experience of the above-mentioned combination study. A phase III study of atezolizumab plus bevacizumab (IMbrave150 study) is ongoing, as is the phase III study of durvalumab plus tremelimumab (HIMALAYA study). The results of these studies are eagerly awaited. 
Kudo: Combination Cancer Immunotherapy with Molecular Targeted

Agents/Anti-CTLA-4 Antibody for Hepatocellular Carcinoma

Table 6. Phase $1 \mathrm{~b}$ of atezolizumab + bevacizumab: baseline characteristics and ORR

\begin{tabular}{lclllll}
\hline & \multicolumn{3}{c}{$\begin{array}{l}\text { ASCO 2018 } \\
(n=23)\end{array}$} & & \multicolumn{2}{l}{$\begin{array}{l}\text { ESMO 2018 } \\
(n=73)\end{array}$} \\
\cline { 2 - 3 } \cline { 5 - 6 } & $n(\%)$ & ORR & & $n(\%)$ & ORR \\
\hline EHS & $11(48)$ & NA & & $51(70)$ & NA \\
MVI & $6(23)$ & NA & & $41(56)$ & NA \\
EHS and/or MVI & $15(65)$ & $11 / 15(73)$ & & $64(88)$ & $18 / 64(28)$ \\
Neither EHS nor MVI & $4(17)$ & $2 / 4(50)$ & & $8(11)$ & $5 / 8(63)$ \\
\hline
\end{tabular}

Investigator-assessed results (RECIST 1.1). ORR, objective response rate; NA, not available; EHS, extrahepatic spread; MVI, macrovascular invasion.

\section{Conclusion}

Prolonged survival and cure may be expected for patients who respond to immune checkpoint inhibitors, as described by Sharma and Allison $[17,18]$. These authors propose that a durable long-lasting response may be achieved with combination immunotherapies in majority of the patients (Fig. 7) [17]. Combination therapies of anti-PD-1/anti-PD-L1 antibodies with other MTAs or with other immune checkpoint inhibitors such as anti-CTLA-4 agents may bring a much better survival in HCC patients and lead to a paradigm shift in the treatment strategy for this disease. The recent development of immune checkpoint inhibitors [19], multiple MTAs [20], and combination therapies as described above [21] may lead to major innovations in the treatment of HCC in the very near future. Further drug development based on an improved understanding of the mechanisms of action of combination therapy should substantially improve the prognosis of patients with unresectable HCC.

\section{Disclosure Statement}

Masatoshi Kudo received lecture fees from Bayer, Eisai, MSD, and Ajinomoto, research grants from Chugai, Otsuka, Takeda, Taiho, Sumitomo Dainippon, Daiichi Sankyo, MSD, Eisai, Bayer, AbbVie, Medico's Hirata, Astellas Pharma, and Bristol-Myers Squibb, and advisory consulting fees from Kowa, MSD, BristolMyers Squibb, Bayer, Chugai, Taiho, Eisai, and Ono Pharmaceutical.

\section{References}

1 El-Khoueiry AB, Sangro B, Yau T, Crocenzi TS, Kudo M, Hsu C, et al. Nivolumab in patients with advanced hepatocellular carcinoma (CheckMate 040): an open-label, non-comparative, phase $1 / 2$ dose escalation and expansion trial. Lancet. 2017 Jun;389(10088):2492-502.

2 Zhu AX, Finn RS, Edeline J, Cattan S, Ogasawara S, Palmer D, et al.; KEYNOTE-224 investigators. Pembrolizumab in patients with advanced hepatocellular carcinoma previously treated with sorafenib (KEYNOTE224): a non-randomised, open-label phase 2 trial. Lancet Oncol. 2018 Jul;19(7):940-52.

3 Postow MA, Chesney J, Pavlick AC, Robert C, Grossmann K, McDermott D, et al. Nivolumab and ipilimumab versus ipilimumab in untreated melanoma. N Engl J Med. 2015 May;372(21):2006-17.

4 Wolchok JD, Kluger H, Callahan MK, Postow MA, Rizvi NA, Lesokhin AM, et al. Nivolumab plus ipilimumab in advanced melanoma. N Engl J Med. 2013 Jul;369(2):122-33.

5 Kelley RK, Abou-Alfa GK, Bendell JC, Kim TY, Borad MJ, Yong WP, Morse M, et al. Phase I/II study of durvalumab and tremelimumab in patients with unresectable hepatocellular carcinoma(HCC): Phase I safety and efficacy analyses. J Clin Oncol. 2017 May;35(15 suppl):4073.

6 Kudo M. Molecular Targeted Agents for Hepatocellular Carcinoma: Current Status and Future Perspectives. Liver Cancer. 2017 Feb;6(2):101-12. 


\begin{tabular}{l|l}
\hline Liver Cancer 2019;8:1-11 \\
\hline DOI: 10.1159/000496277 & $\begin{array}{l}\text { @ 2019 S. Karger AG, Basel } \\
\text { www.karger.com/lic }\end{array}$ \\
\hline
\end{tabular}

Kudo: Combination Cancer Immunotherapy with Molecular Targeted

Agents/Anti-CTLA-4 Antibody for Hepatocellular Carcinoma

7 Chen DS, Mellman I. Oncology meets immunology: the cancer-immunity cycle. Immunity. 2013 Jul 25;39(1): $1-10$.

8 Wing K, Onishi Y, Prieto-Martin P, Yamaguchi T, Miyara M, Fehervari Z, et al. CTLA-4 control over Foxp3+ regulatory T cell function. Science. 2008 Oct;322(5899):271-5.

9 Voron T, Marcheteau E, Pernot S, Colussi O, Tartour E, Taieb J, et al. Control of the immune response by proangiogenic factors. Front Oncol. 2014 Apr; 4:70.

10 Tiegs G, Lohse AW. Immune tolerance: what is unique about the liver. J Autoimmun. 2010 Feb;34(1):1-6.

11 Kato T, Bao X, Macgrath S, Tabata K, Hori Y, Tachino S, et al. Lenvatinib mesilate (LEN) enhanced antitumor activity of a PD-1 blockade agent by potentiating Th1 immune response. Ann Oncol. 2016;27(suppl 6):2PD.

12 Ikeda M, Sung MW, Kudo M, Kobayashi M, Baron AD, Finn RS, et al. A phase $1 \mathrm{~b}$ trial of lenvatinib (LEN) plus pembrolizumab (PEM) in patients (pts) with unresectable hepatocellular carcinoma (uHCC). J Clin Oncol. 2018;36(15 suppl):4076.

13 Stein S, Pishvaian MJ, Lee MS, Lee KH, Hernandez S, Kwan A, et al. Safety and clinical activity of 1L atezolizumab + bevacizumab in a phase Ib study in hepatocellular carcinoma (HCC). J Clin Oncol. 2018 May;36(15 suppl); 4074.

14 Xu JM, Zhang Y, Jia R, Wang Y, Liu R, Zhang G, et al. Anti-programmed death-1 antibody SHR-1210 (S) combined with apatinib (A) for advanced hepatocellular carcinoma (HCC), gastric cancer (GC) or esophagogastric junction (EGJ) cancer refractory to standard therapy: A phase 1 trial. J Clin Oncol. 2018 May;36(15 suppl); 4075.

15 Pishvaian MJ, Lee MS, Ryoo BY, Stein S, Lee KH, Verret W, et al. Updated safety and clinical activity results from a Phase Ib study of atezolizumab + bevacizumab in hepatocellular carcinoma (HCC). ESMO 2018; Munich; October 19-12. LBA26.

16 Yamashita T, Kudo M, Ikeda K, Izumi N, Ikeda M, Okusaka T, et al. Analysis of Japanese subpopulation from reflect trial. 18th Annu Meet Japan Assoc Mol Targeted Ther HCC; Tokyo; July 14, 2018.

17 Sharma P, Allison JP. The future of immune checkpoint therapy. Science. 2015 Apr;348(6230):56-61.

18 Sharma P, Allison JP. Immune checkpoint targeting in cancer therapy: toward combination strategies with curative potential. Cell. 2015 Apr;161(2):205-14.

19 Kudo M. Immune checkpoint blockade in hepatocellular carcinoma: 2017 update. Liver Cancer. 2016 Nov; 6(1):1-12.

20 Kudo M, Finn RS, Qin S, Han KH, Ikeda K, Piscaglia F, et al. Lenvatinib versus sorafenib in first-line treatment of patients with unresectable hepatocellular carcinoma: a randomised phase 3 non-inferiority trial. Lancet. 2018 Mar;391(10126):1163-73.

21 Kudo M. Combination Cancer Immunotherapy in Hepatocellular Carcinoma. Liver Cancer. 2018 Mar;7(1): 20-7. 